\title{
CONHECIMENTO DE GESTANTES DO PAPEL DO ALEITAMENTO MATERNO NO SISTEMA ESTOMATOGNÁTICO
}

\author{
KNOWLEDGE OF PREGNANT WOMEN ABOUT THE IMPORTANCE OF \\ BREASTFEEDING FOR THE STOMATOGNATHIC SYSTEM
}

\section{Silvana Marchiori de ARAÚJ0'; Viviani Aparecida Paim da Silva DUARTE²; Eliane Garcia da SILVEIRA3; Maria Mercês Aquino Gouveia FARIAS; Beatriz Helena Eger SCHMITT ${ }^{4}$}

1 - Doutorado em Odontopediatria. Professora de Clínica Integrada Infantil e Materno Infantil.

Centro de Ciências da Saúde, Universidade do Vale do Itajaí-SC;

2 - Graduada em Odontologia. Universidade do Vale do Itajaí-SC;

3 - Mestrado em Odontopediatria. Professora de Clínica Integrada Infantil e Materno Infantil.

Centro de Ciências da Saúde, Universidade do Vale do Itajaí-SC;

4 - Mestrado em Odontopediatria. Professora de Clinica Integrada Infantil e Materno Infantil.

Centro de Ciências da Saúde, Universidade do Vale do Itajaí-SC.

\section{RESUMO}

Objetivo: avaliar o conhecimento de um grupo de gestantes em relação à importância do aleitamento materno sob o aspecto odontológico. Material e método: a população-alvo foi composta por gestantes em atendimento de pré-natal na Unidade de Saúde Clínica Materno Infantil Dra Maria da Graça, no Município de Caçador-SC. A amostra foi do tipo não probabilística obtida por conveniência. $\mathrm{O}$ instrumento para coleta de dados foi um questionário constituído por duas partes: na primeira foram coletadas informações para caracterização das participantes; na segunda foi investigado o conhecimento das gestantes sobre a importância do aleitamento materno sob o aspecto odontológico com questões dicotômicas que abordaram o aleitamento materno e sua relação com o desenvolvimento do sistema estomatognático. Para a determinação do conhecimento da população, foram definidos três níveis, a saber: Baixo: para um total de acertos entre 1 e 3 questões; Médio: para um total de acertos entre 4 e 7 questões; Bom: para um total de acertos entre 8 e 11 questões. $\mathrm{O}$ tratamento estatístico foi do tipo descritivo, mediante cálculo da frequência relativa. Para analisar a relação entre o conhecimento das gestantes com a escolaridade e renda familiar, foi aplicado o teste Qui-Quadrado. Resultados: observou-se que $60 \%$ das gestantes apresentaram nível bom de conhecimento, 36\% nível médio e $4 \%$ das participantes com nível baixo de conhecimento. Conclusão: a maioria das gestantes apresentou nível de conhecimento bom em relação à importância do aleitamento materno sob o aspecto odontológico; o conhecimento das gestantes não apresentou relação estatisticamente significante com o nível de escolaridade e a renda familiar.

PALAVRAS-CHAVE: Aleitamento Materno; Sistema Estomatognático; Saúde Bucal.

\section{INTRODUÇÃO}

O desenvolvimento adequado das habilidades orais, definidas como a resposta motora oral durante a alimentação, é fundamental para a criança. Problemas na movimentação das estruturas ou funções do sistema estomatognático durante a amamentação ou quando da oferta de outros alimentos podem comprometer não apenas o desenvolvimento motor oral da criança, mas também ter repercussões importantes sobre a nutrição infantil ${ }^{1}$.

$O$ aleitamento materno é foco de estudos de interesse multiprofissional por seu valor nutricional, e imunológico e também por estimular o contato físico e a interação entre mãe e filho, o que colabora para o desenvolvimento biopsicossocial da criança $^{2}$. Também, a intensa atividade muscular que proporciona promove o desenvolvimento craniofacial, favorecendo o adequado selamento labial durante o estado de repouso oral, que estimula a correção do retrognatismo mandibular fisiológico e posicionando corretamente a língua na região palatina dos dentes incisivos centrais. Essas vantagens justificam a recomendação do aleitamento materno exclusivo durante os primeiros seis meses de vida ${ }^{1,3}$.

A influência da amamentação no desenvolvimento de má-oclusão foi estudada por diversos autores, os quais concluíram que o exercício da amamentação é favorável à manutenção de uma boa relação oclusal, pois permite o correto desenvolvimento do sistema estomatognático do bebê $\hat{e}^{4,5}$.

Portanto, diante do exposto, o presente estudo teve como objetivo avaliar o nível de conhecimento de gestantes em atendimento de pré-natal na Unidade de Saúde Clínica Materno Infantil Dra Maria da Graça, no Município de Caçador-SC, em relação à importância do aleitamento materno sob o aspecto odontológico dando ênfase aos danos que podem causar no sistema estomatognático. 


\section{MATERIAIS E MÉTODOS}

Este estudo foi previamente aprovado pelo Comitê de Ética em Pesquisa da Universidade do Vale do Itajaí (UNIVALI) sob o parecer $n^{\circ} 1.311 .093$. Trata-se de uma pesquisa de natureza descritiva do tipo transversal mediante coleta de dados primários.

A população-alvo da pesquisa foi constituída por gestantes em atendimento de pré-natal na Unidade de Saúde Clínica Materno Infantil Dra Maria da Graça, no Município de Caçador-SC, durante o $1^{\circ}$ e $2^{\circ}$ semestres de 2016. A amostra foi do tipo não probabilístico obtida por conveniência, isto é, integraram a pesquisa todas as gestantes que por livre e espontânea vontade aceitaram participar da pesquisa, assinando o Termo de Consentimento Livre e Esclarecido.

O instrumento para coleta de dados foi um questionário constituído por duas partes. A primeira continha cinco itens para caracterização sociodemográfica da amostra que incluiu idade, escolaridade e renda familiar. Enquanto que, a segunda apresentava onze questões do tipo fechado, dicotômicas, abordando aspectos como: duração do aleitamento materno; relação do aleitamento materno com respiração bucal; instalação de hábitos de sucção deletérios e desenvolvimento orofacial da criança e leite materno como fator etiológico de cárie dental, conforme Quadro 1.

Para classificar o conhecimento das participantes, definiu-se uma escala, na qual constam três níveis classificatórios, a saber: Baixo, para um total de acertos entre 1 e 3 questões; Médio, para

Quadro 1 - Questões do instrumento de coleta de dados

\begin{tabular}{l}
\hline QUESTÕES \\
\hline CERTO 0 tempo ideal de aleitamento materno exclusivo \\
é até os 6 meses de idade \\
Q2- 0 tempo recomendado de aleitamento materno é \\
até os dois anos de idade
\end{tabular}

Q3- Criança que mama no peito durante o tempo ideal tem menor probabilidade de chupar dedo ou chupeta

Q4- 0 aleitamento materno promove o crescimento da mandíbula, fazendo com que esta se encontre em posição ideal no momento de erupção dos dentes de leite

Q5- 0 exercício muscular durante a sucção do seio ajuda na prevenção de problemas de posicionamento incorreto dos dentes

Q6- 0 aleitamento materno estimula a respiração nasal

\section{Q7- 0 aleitamento materno previne deglutição atípica}

Q8- 0 aleitamento materno previne a colocação incorreta da língua dentro da boca, o que desencadearia dificuldades para pronunciar determinadas palavras

Q9- 0 exercício desenvolvido pelo aleitamento materno tem participação no crescimento dos ossos da face e dos músculos da região oral

Q10- 0 leite materno pode causar cárie dental nas crianças

Q11- A cárie dental pode ser evitada

Fonte: Autoria própria (2016). um total de acertos entre 4 e 7 questões e Bom, para um total de acertos entre 8 e 11 questões.

As respostas emitidas foram tabuladas e organizadas com o auxílio do programa Microsoft Office Excel 2010. O tratamento estatístico foi do tipo descritivo, mediante cálculo da frequência relativa. Para as questões do domínio cognitivo, foram efetivadas duas análises. Primeiro, obteve-se o índice de acertos e erros para cada uma das onze questões que constituem parte do instrumento de coleta de dados, após foi calculada a frequência de sujeitos para cada um dos níveis de conhecimento definidos para este estudo. Para analisar a relação entre o conhecimento das gestantes e renda e escolaridade, foi aplicado o teste Qui-Quadrado $(p \leq 0,05)$.

\section{RESULTADO}

A amostra do estudo foi constituída por 50 gestantes, e a caracterização da amostra pode ser observada na Tabela 1.

Na Figura 1, pode-se observar a distribuição da frequência relativa das gestantes quanto ao grau de conhecimento.

A distribuição da frequência relativa das gestantes segundo o grau de conhecimento em função da renda familiar e nível de escolaridade pode-se observar nas Figuras 2 e 3.

Pelo teste Qui-Quadrado não foi observada relação estatisticamente significante entre o conhecimento das gestantes com a renda ( $p=0,08159081)$, e escolaridade $(p=0,574266938)$.

Na Figura 4, visualiza-se a frequência relativa das respostas corretas e incorretas emitidas pelas participantes em cada uma das questões formuladas no estudo.

Tabela 1- Caracterização da amostra, segundo escolaridade, idade e renda familiar

\begin{tabular}{lll}
\hline & & $\mathrm{N} \%$ \\
\cline { 2 - 3 } & Escolaridade das mães & \\
& Ensino Fundamental & $27,54 \%$ \\
& Ensino Médio & $19,38 \%$ \\
& Ensino Superior & $4,8 \%$ \\
\cline { 2 - 3 } Caracterização & Idade das mães (anos) & \\
da Amostra & Até 20 & $13,26 \%$ \\
& De 21 a 30 & $26,52 \%$ \\
& De 31 a 41 & $11,22 \%$ \\
\cline { 2 - 3 } & Renda familiar (Salários mínimos) & \\
& Até 3 & $46,92 \%$ \\
& Mais de 3 até 5 & $3,6 \%$ \\
& Mais de 5 & $1,2 \%$ \\
\hline
\end{tabular}

Fonte: Autoria própria (2016)

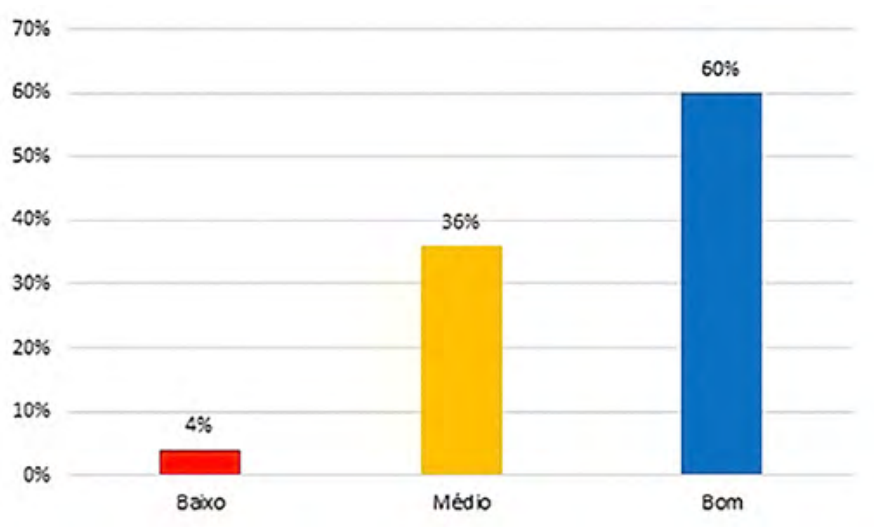

Figura 1 - Distribuição da frequência relativa da classificação das gestantes segundo o grau de conhecimento 
As letras $Q$ acompanhadas dos números de 1 a 11 representam as afirmativas descritas abaixo:

Q1- O tempo ideal de aleitamento materno exclusivo é até os 6 meses de idade.

Q2- O tempo recomendado de aleitamento materno é até os dois anos de idade.

Q3- Criança que mama no peito durante o tempo ideal tem menor probabilidade de chupar dedo ou chupeta.

Q4- O aleitamento materno promove o crescimento da mandíbula, fazendo com que esta se encontre em posição ideal no momento de erupção dos dentes de leite.

Q5- O exercício muscular durante a sucção do seio ajuda na prevenção de problemas de posicionamento incorreto dos dentes.

Q6- O aleitamento materno estimula a respiração nasal.

Q7- O aleitamento materno previne deglutição atípica.

Q8- O aleitamento materno previne a colocação incorreta da língua dentro da boca, o que desencadearia dificuldades para pronunciar determinadas palavras.

Q9- O exercício desenvolvido pelo aleitamento materno tem participação no crescimento dos ossos da face e dos músculos da região oral.

Q10- O leite materno pode causar cárie dental nas crianças.

Q11- A cárie dental pode ser evitada.

\section{DISCUSSÃO}

Do ponto de vista odontológico, o aleitamento materno é importante para o desenvolvimento do sistema estomatognático, pois a criança recebe vários estímulos que proporcionam o seu desenvolvimento físico e psicológico. Os estímulos são tátil-cinestésicos, térmicos, olfativos, visuais, auditivos e motores e possibilitarão o desenvolvimento das funções básicas de sucção, mastigação, deglutição e respiração ${ }^{6-8}$.

Quando analisadas as questões individualmente, observa-se que a maioria das gestantes responderam corretamente todas as questões.

A respeito do tempo ideal da amamentação exclusiva, as gestantes afirmaram que deve ser de seis meses, o que é recomendado pela Organização Mundial da Saúde (OMS) ${ }^{9}$.

Sobre o tempo total de amamentação, as gestantes mostraram conhecimento, afirmando que o tempo ideal seria até os dois anos de idade, concordando com a recomendação da Organização Mundial da Saúde (OMS) ${ }^{9}$ e o Ministério da Saúde do Brasil ${ }^{10}$ que diz ser ideal o aleitamento materno exclusivo por seis meses e complementado até os dois anos ou mais. Resultado diferente foi encontrado por Guimarães et al. ${ }^{11}$ (2012), onde somente trinta por cento das participantes responderam corretamente este item.

O aleitamento materno é considerado indispensável nos seis primeiros meses de vida da criança, tanto para seu desenvolvimento físico como emocional, sendo que previne a instalação dos hábitos bucais deletérios e promove o crescimento e desenvolvimento normal das estruturas da face ${ }^{12}$. Não podendo ser desconsiderada a importância do aleitamento materno no desenvolvimento psicológico da criança, pois esta prática é fundamental para a troca afetiva, como também para as experiências de contato social, e que poderá influenciar na personalidade desta criança futuramente ${ }^{13-15}$.

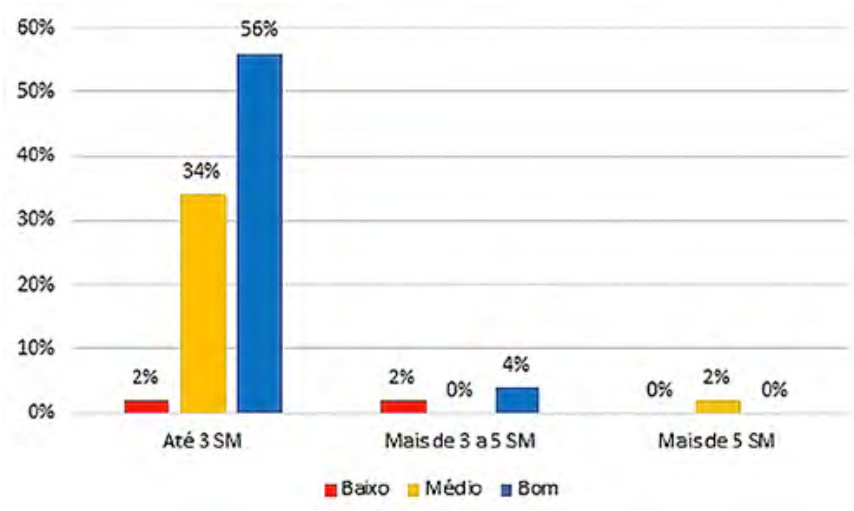

Figura 2 - Distribuição da frequência relativa das gestantes segundo grau de conhecimento em função da renda familiar

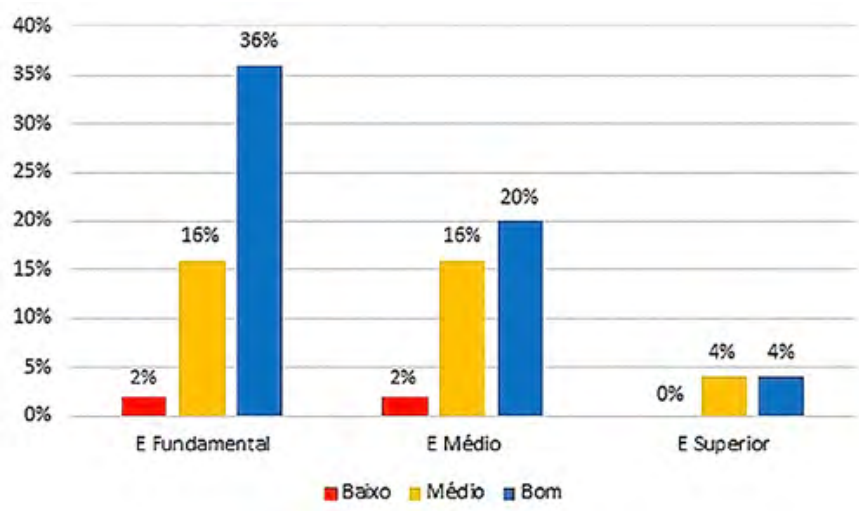

Figura 3 - Distribuição da frequência relativa das gestantes, segundo grau de conhecimento em função da escolaridade

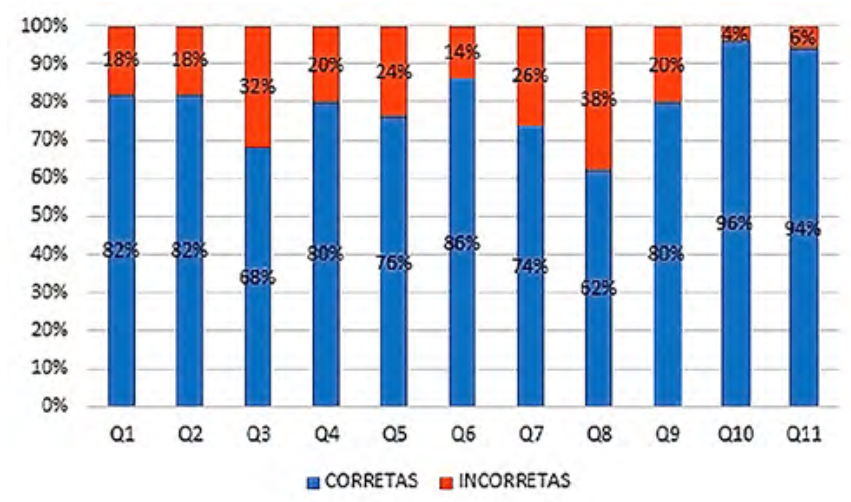

Figura 4 - Distribuição da frequência relativa das respostas emitidas pelas gestantes para cada uma das questões do estudo 
O desmame precoce também pode acarretar a diminuição do desenvolvimento oro-motor adequado, podendo provocar alterações na postura, prejudicando as funções de mastigação, deglutição, respiração e articulação dos sons da fala ${ }^{16,17}$.

O exercício realizado durante a sucção de rebaixamento e elevação da mandíbula concomitantes, modificam a relação maxilo-mandibular do bebê propiciando o crescimento ósseo-mandibular adequado. Com esse crescimento, ocorre uma ampliação do espaço bucal e melhor arranjo dos germes dentários dentro do osso alveolar, contribuindo para a acomodação e livre movimentação da língua dentro da cavidade bucal ${ }^{18}$.

De todas as questões levantadas, a que teve maior percentual de respostas incorretas foi sobre a importância do aleitamento materno para o posicionamento correto da língua, que favorece o desenvolvimento da fala, evitando dificuldades para pronunciar determinadas palavras.

A sucção da mama durante o aleitamento materno tem papel fundamental no desenvolvimento Oro-motor adequado, pois estimula os movimentos e funções dos órgãos fono-articulatórios (OFAs): lábios; língua, mandíbula; maxila, bochechas; palato mole; palato duro; soalho da boca; musculatura oral e arcadas dentárias ${ }^{19}$. Dessa forma, promove o correto desenvolvimento da musculatura facial e das demais estruturas do sistema estomatognático ${ }^{20}$. O principal estímulo ao crescimento orofacial é a amamentação, pois através dela, toda a musculatura da metade inferior da face é estimulada, proporcionando o crescimento da estrutura óssea mandibular. Durante o aleitamento materno, a tonicidade da musculatura facial do bebê aumenta, o que contribui para evitar a síndrome do respirador bucal. Há evidências de que o aleitamento materno é um fator preventivo da síndrome do respirador bucal ${ }^{21,22}$. A amamentação traz benefícios nutricionais, imunológicos, emocionais, além de ser fundamental para o estabelecimento do padrão respiratório adequado na criança ${ }^{23}$.

Apesar da maioria das gestantes ter respondido corretamente a questão a respeito da criança que mama no peito durante o tempo ideal, ter menor probabilidade de chupar dedo ou chupeta, ao mesmo tempo foi a questão que apresentou o segundo maior percentual de respostas incorretas com mais de trinta por cento.

As crianças que não são amamentadas ao seio possuem maior probabilidade de introduzir o dedo na boca, sendo que necessitam exercitar a musculatura, pois sua satisfação nutricional é mais rápida. Ao contrário, as crianças amamentadas no peito possuem menor probabilidade de desenvolver hábitos bucais deletérios no decorrer de suas vidas em razão de um intenso trabalho muscular realizado para a sucção do leite materno, o que gera uma musculatura perioral fatigada, evitando, assim, que busquem outro tipo de sucção (como dedo, chupeta ou objetos) a fim de se satisfazerem nutricional e emocionalmente, pois sua fome neural já foi saciada ${ }^{2,45,13,15,24-26}$.

Vários autores sugerem que a sucção não-nutritiva pode ser responsável pelo surgimento de más oclusões na infância, principalmente mordida aberta anterior, overjet e mordida cruzada posterior $^{6-8}$. A amamentação diminui o risco de más oclusões ${ }^{27}$.

Mais de noventa por centos das gestantes deste estudo acreditam que o leite materno pode causar cárie dental nas crianças, no entanto a literatura revela controvérsia. $\mathrm{O}$ leite materno tem sido citado na literatura como responsável pelo aparecimento de lesões cariosas na primeira infância, porém, muitos estudos defendem sua não cariogenicidade ${ }^{28,29}$.

Resultados diferentes destes foram encontrados por outros estudos $^{30-33}$, onde a maioria das entrevistadas acredita que o leite materno não é cariogênico,

Não há evidências que comprovem que o leite materno está associado com o surgimento da cárie dentária e que esta relação é complexa e confundida com muitas variáveis, principalmente infecção por Streptococcus mutans, hipoplasia de esmalte, ingestão de açúcares, em suas mais variadas formas e condições sociais, representadas pela educação e nível socioeconômico dos pais s $^{28,34}$.

Uma das questões com o maior percentual de acerto foi a questão que perguntava se é possível evitar a cárie dentária, quase a totalidade das participantes responderam que sim, semelhante ao encontrado no estudo de Besen e Silva ${ }^{30}$ (2015) e Becker e Sampaio ${ }^{31}$ (2014). A cárie dentária pode ser prevenida, controlada ou mesmo revertida. Para prevenção, é necessário conhecer sua etiologia e os fatores de risco para o seu desenvolvimento $^{35}$. A medida mais adotada para se prevenir a cárie é a escovação dentária ${ }^{36}$.

Neste estudo, quando analisado, o número de acertos de todas as participantes e classificadas quanto ao nível de conhecimento do aleitamento sob o aspecto odontológico, observou-se que a maioria das participantes apresentou nível bom de conhecimento, diferente dos estudos de Guimarães et al. ${ }^{11}$ (2012) e Massoni et al..$^{37}$ (2010), porém semelhante ao estudo de Besen e Silva ${ }^{30}$ (2015).

Pelo teste Qui-Quadrado não foi observado relação estatisticamente significante entre o conhecimento das gestantes e a renda e escolaridade, semelhante a outros estudos ${ }^{16,30,31,38}$

A Estratégia Saúde da Família é um modelo adotado pelo Ministério da Saúde para estruturação da Atenção Primária com o objetivo de reverter o modelo assistencial, $80 \%$ dos problemas de Saúde podem ser resolvidos nesse nível de atenção. Portanto, o Cirurgião-Dentista deve dar ênfase ao aleitamento materno como preventivo de aspectos relevantes da promoção de saúde do sistema estomatognático, que desempenha as funções de respiração, deglutição, mastigação e fala, podendo acarretar alterações orais de relevância como as más oclusões.

Os profissionais da fonoaudiologia e da odontologia estão devidamente capacitados para orientar nos aspectos relacionados ao sistema estomatognático, o que pode diminuir os problemas de fonação, oclusão dentária, respiração, motricidade orofacial e deglutição, e, inseridas em ações de equipes interdisciplinares capacitadas e treinadas, são mais eficazes em promover a saúde materno-infantil ${ }^{39}$.

\section{CONCLUSÃO}

a. De acordo com os achados concluiu-se que:

b. A maioria das gestantes apresentou bom nível de conhecimento em relação à importância do aleitamento materno sob o aspecto odontológico.

c. Não foi observada relação estatisticamente significante entre o conhecimento das gestantes com o nível de escolaridade e a renda familiar.

\section{AGRADECIMENTOS}

Ao Programa de Iniciação Científica Artigo170/Governo do Estado de Santa Catarina/ Vice-Reitoria de Pesquisa, 
Pós-Graduação, Extensão e Cultura da Universidade do Vale do Itajaí - UNIVALI, que financiaram a pesquisa (Edital ProPPEC/ UNIVALI 02/2016).

\section{REFERÊNCIAS}

01. Silveira LM, Prade LS, Ruedell AM, Haeffner LSB, Weinmann ARM. Aleitamento materno e sua influência nas habilidades orais de crianças. Rev. Saúde Coletiva. 2013; 47(1): 37-43.

02. Albuquerque SSL, Duarte RC, Cavalcanti AL, Beltrão EM. A influência do padrão de aleitamento no desenvolvimento de hábitos de sucção não nutritivos na primeira infância. Cienc. Saude Coletiva. 2010; 15(2): 371-378.

03. Santana JM, Brito SM, Santos DB. Amamentação: conhecimento e prática de gestantes. O Mundo da Saúde. 2013; 37(3): 259-267.

04. Fonseca-Machado MO, Paula MSR, Parreira BDM, Stefanello J, Gomes-Sponholz F. Comparação do conhecimento sobre aleitamento materno entre mulheres no período pós-parto. Rev. Enferm. UERJ. 2013; 21(1): 66-77.

05. Carvalho CM, Carvalho LFPC, Forte FDS, Aragão MS, Costa LJ. Prevalência de mordida aberta anterior em crianças de 3 a 5 anos em cabedelo/PB e relação com hábitos bucais deletérios. Pesq. Bras. Odontoped. Clin. Integr. 2009; 9(2): 205-210.

06. Cota JB. Vantagens do aleitamento materno para o desenvolvimento do sistema estomatognático [Monografia de Especialização). Governador Valadares: Curso de Especialização Atenção Básica em Saúde da Família; Universidade Federal de Minas Gerais; 2011.

07. Ferreira FV, Marchionatti AM, Oliveira MDM, Praetzel JR. Associação entre a duração do aleitamento materno e sua influência sobre o desenvolvimento de hábitos orais deletérios. RSBO. 2010; 7(1):35-40.

08. Moimaz SAS, Rocha NB, Garbin AJI, Saliba O. O aleitamento materno e sua relação com a prevenção das oclusopatias. Rev. Bras. Pesq. Saúde. 2012: 14(4): 29-35.

09. World Health Organization (WHO). Breastfeeding [Internet]. [Acessado em 11 jul 2020]. Disponível em: https://www.who.int/ health-topics/breastfeeding\#tab=tab_2.

10. Brasil. Ministério da Saúde. Secretaria de Atenção à Saúde. Departamento de Atenção Básica. Saúde da criança: aleitamento materno e alimentação complementar. Ministério da Saúde, Secretaria de Atenção à Saúde, Departamento de Atenção Básica. 2. ed. Brasília: Ministério da Saúde; 2015. 184 p. (Cadernos de Atenção Básica, n. 23).

11. Guimarães LAOP, Marçal F, Zuffi FB, Fonseca-Machado FO. Pet-Saúde na identificação do conhecimento de gestantes sobre aleitamento materno. Cienc. Cuid. Saude. 2012; 11(3): 454-462.

12. Gisfrede TF, Kimura JS, Reyes A, Bassi J, Drugowick R, Matos R, et al. Hábitos bucais deletérios e suas consequências em Odontopediatria. Rev. Bras. Odontol. 2016; 73(2): 144-149.

13. Antunes LS, Antunes LAA, Corvino MPF, Maia LC. Amamentação natural como fonte de prevenção em saúde. Ciênc. Saúde Coletiva. 2008; 13(1): 103-109.

14. Massuia JM, Carvalho WO, Matsuo T. Má Oclusão, Hábitos Bucais e Aleitamento Materno: Estudo de Base Populacional em um Município de Pequeno Porte. Pesq Bras Odontoped Clin Integr. 2011; 11(3): 451-57.

15. Pellizzaro D, Cota ALS, Contreras EFR, Correa GO, Garbelini CCD. Aleitamento Natural e sua Relação com o Sistema Estomatognático. UNOPAR Cient. Ciênc. Biol. Saúde, 2008; 10(2): 63-68.

16. Medeiros UV; Knupp RRS. Análise de conhecimentos e práticas de mães sobre saúde bucal. Rev. bras. Pesqui. Saúde. 2010; 12(4): 13-18.
17. Neiva FCB, Cattoni DM, Ramos JLA, Issler H. Desmame precoce: implicações para o desenvolvimento motor-oral. J. Pediatr. 2003; 79(1): 7-12.

18. Guedes-Pinto AC. Odontopediatria. São Paulo: Santos; 2010. 1048 p.

19. Sanches MTC. Manejo clínico das disfunções orais na amamentação. J Pediatr. 2004; 80(5): 155-162.

20. Trawitzki LVV, Anselmo-Lima VT, Melchior MO, Grechi TH, Valera FCP. Aleitamento e hábitos orais deletérios em respiradores orais e nasais. Rev Bras Otorrinolaringol. 2005; 71(6): 747-751.

21. Moimaz SAS, Rocha NB, Garbin AJI, Saliba O. Relação entre aleitamento materno e hábitos de sucção não nutritivos. Ciência \& Saúde Coletiva. 2011; 16(5): 2477-2484.

22. Neiva FCB, Cattoni DM, Ramos JLA, Issler H, Desmame precoce: implicações para o desenvolvimento motor-oral. J. Pediatr. 2003; 79(1): 7-12

23. Goulardins JB. O aleitamento materno e a síndrome do respirador oral.[online] Revista Digital. 2010; 15(150).

24. Bernardes D, Kusminskas M, Santos DF, Grangeiro RSF, Lopes MGO. Associação entre duração do aleitamento materno e sua influência sobre o desenvolvimento de hábitos orais deletérios. Rev. UniVap (online). 2016; 22(40): 35-40.

25. Emmerich A, Fonseca L, Elias AM, Medeiros UV. Relação entre hábitos bucais, alterações oronasofaringianas e mal-oclusões em préescolares de Vitória, Espírito Santo, Brasil. Cad. Saúde Pública. 2004; 20(3): 689-697.

26. Miotto MHMB, Caxias FP, Campos DMKS, Ferreira LFPE, Barcellos LA. Aleitamento materno como fator de proteção contra a instalação de hábitos bucais deletérios. Rev. CEFAC. 2014; 16(1): 244-251.

27. Peres KG, Cascaes AM, Nascimento GG, Victora CG. Effect of breastfeeding on malocclusions: a systematic review and metaanalysis. Acta Paediatr. 2015; 104(467): 54-61.

28. Lemos LVFM, Correia MF, Spolidório DMP, Myaki SI, Zuanon ACC. Cariogenicidade do Leite Materno: Mito ou Evidência Científica. Pesq. Bras. Odontoped. Clin. Integr. 2012; 12(2): 273-78.

29. Mohebbi SZ, Virtanen JI, Vahid Golpayegani M, Vehkalahti MM. Feeding habits as determinants of early childhood caries in a population where prolonged breastfeeding is the norm. Community Dent. Oral Epidemiol. 2008; 36(4): 363-69.

30. Besen LW, Silva MM. Aleitamento materno sob o aspecto odontológico [Trabalho de Conclusão de Curso de Graduação]. Itajaí: Curso de Odontologia da Universidade do Vale do Itajaí; 2015.

31. Becker AD, Sampaio MB. Cárie de acometimento precoce: conhecimento de pais e responsáveis [Trabalho de Conclusão de Curso de Graduação]. Itajaí; Curso de Odontologia da Universidade do Vale do Itajaí; 2014.

32. Carlin MC, Seidel S. Cárie de acometimento precoce: visão de mães de crianças de 0 a 4 anos de idade [Trabalho de Conclusão de Curso de Graduação]. Itajaí: Curso de Odontologia da Universidade do Vale do Itajaí; 2015.

33. Franzin LCS, Bijella MFTB. Conhecimento de um grupo de mães sobre a saúde bucal de seus filhos. JBP Rev. Ibero Am. Odontopediatr. Odontol. Bebê. 2005: 8(45): 333-341.

34. Ribeiro NEM, Ribeiro MAS. Aleitamento materno e cárie do lactente e do pré-escolar: uma revisão crítica. J. Pediatr. 2004; 80(5): 199-210.

35. Losso EM, Tavares MCR, Silva JYB, Urban CA. Cárie precoce e severa na infância: uma abordagem integral. J. Pediatr. 2009; 85(4): 295-300.

36. Massoni ACLT, Ferreira JMS, Silva FDSCM, Carvalho LFPC, Duarte RC. Conhecimento de Gestantes sobre a Saúde Bucal dos Bebês. Rev. Bras. Ciênc. Saúde. 2009; 13(1): 41-47. 
37. Massoni ACLT, Paulo SF, Forte FDS, Freitas CHSM, Sampaio FC. Saúde bucal infantil: conhecimento e interesse de pais e responsáveis. Pesq. Bras. Odontoped. Clin. Integr. 2010; 10(2): 321-325.

38. Theodoro DS, Gigliotti MP, Oliveira TM, Silva SMB, Machado MAAM. Fator socioeconômico o grau de conhecimento das mães em relação à saúde bucal de bebês. Odontol. Clín. Cient. 2007; 6(2): 133-137.

39. Maahs MAP, Kurtz L, Bonamigo AW, Tamanini AS. Promoção do aleitamento materno em um contexto interdisciplinar. Rev. de Atenção à Saúde. 2015; 13(43): 46-52.

\section{ABSTRACT}

Aim: to evaluate the knowledge of a group of pregnant women about the importance of breastfeeding under the dentistry aspect. Material and method: the target population was composed of pregnant women under prenatal care at the "Dra Maria da Graça" Mother and Child Clinical Health Unit of the municipality of Caçador-SC. Sample was non-probabilistic and obtained by convenience. Data collection instrument was a questionnaire consisting of two parts: in the first part, information was collected to characterize participants; in the second part, the knowledge of pregnant women about the importance of breastfeeding under the dentistry aspect was investigated with dichotomous questions that addressed breastfeeding and its relationship with the development of the stomatognathic system. Three levels were defined to determine the knowledge of participants: Low - for total number of correct answers between 1 and 3 questions; Medium: for total number of correct answers between 4 and 7 questions; Good: for total of 8 to 11 questions. Statistical treatment was of descriptive type, by calculating the relative frequency. To analyze the relationship between knowledge of pregnant women with schooling and family income, the Chi-Square test was applied. Results: it was observed that $60 \%$ of pregnant women had good level of knowledge, $36 \%$ had medium level and $4 \%$ of participants had low level of knowledge. Conclusion: most pregnant women had good level of knowledge regarding the importance of breastfeeding under the dentistry aspect; the knowledge of pregnant women did not show statistically significant relationship with schooling and family income.

KEYWORDS: Breastfeeding; Stomatognathic System; Oral Health.

\section{AUTOR PARA CORRESPONDÊNCIA}

Silvana Marchiori de Araújo

Rua Uruguai, 458, Centro, Itajaí - SC, Brasil, Cep.: 88302-901

E-mail: silmarchiori@univali.br 